\title{
VARIATIONAL PRINCIPLES AND CONSERVATION LAWS IN THE DERIVATION OF RADIATION BOUNDARY CONDITIONS FOR WAVE EQUATIONS
}

\author{
EDWIN F. G. VAN DAALEN, JAN BROEZE, AND EMBRECHT VAN GROESEN
}

\begin{abstract}
Radiation boundary conditions are derived for partial differential equations which describe wave phenomena. Assuming the evolution of the system to be governed by a Lagrangian variational principle, boundary conditions are obtained with Noether's theorem from the requirement that they transmit some appropriate density - such as the energy density - as well as possible.

The theory is applied to a nonlinear version of the Klein-Gordon equation. For this application numerical test results are presented. In an accompanying paper the theory is applied to a two-dimensional wave equation.
\end{abstract}

\section{INTRODUCTION}

In this paper it is shown how variational methods and conservation laws can be used to derive radiation (or absorbing) boundary conditions for partial differential equations which describe wave phenomena. Such boundary conditions are desirable in order to limit the size of the computational domain and thus computation time and data storage as much as possible, while minimizing the effects of reflected waves on the solution in the interior of the domain.

Many methods for developing radiation boundary conditions have been used. For wave equations, the boundary conditions proposed by Bayliss and Turkel [1, 2], Engquist and Halpern [6], Engquist and Majda [7, 8], and Higdon [12, $13,14]$ are well known. These boundary conditions have in common that they are based on (properties of) solutions to the partial differential equations under consideration.

In this paper, radiation boundary conditions are derived without the assumption that solutions are available beforehand, and therefore they are applicable to nonlinear and dispersive systems too. Moreover, these boundary conditions render the problem well-posed in the sense that the integral of some appropriate density (e.g., the energy density) over the computational domain does not increase.

Received June 21, 1990; revised January 9, 1991.

1991 Mathematics Subject Classification. Primary 35L05, 35L65; Secondary 65M99, 65 N99.

Key words and phrases. Radiation boundary conditions, variational principles, conservation laws, wave equations.

The first two authors are grateful to Professor Zandbergen (University of Twente) for his helpful advice and encouragement. These investigations were supported by the Netherlands Technology Foundation (STW) and the National Foundation for Computer Facilities (NCF). 
The outline of this paper is as follows. In $\S 2$ we develop a theory of radiation boundary conditions for continuous systems described by a Lagrangian density. This theory is illustrated in $\S 3$ with explicit boundary conditions for a nonlinear version of the Klein-Gordon equation. Numerical test results for this application are presented and discussed in $\S 4$. The reader is referred to the accompanying paper [5] for an application of the theory, including test results, to a two-dimensional wave equation.

\section{RADIATION BOUNDARY CONDITIONS FOR CONTINUOUS SYSTEMS}

Consider a continuous system described by the variable $u$, which is a function of the spatial coordinates $\vec{x}=\left(x_{1}, x_{2}, \ldots, x_{n}\right)$ and the time $t$.

It is assumed that the evolution of the system can be derived from a variational principle. With a Lagrangian density $\mathscr{L}$, an expression in $\vec{x}, t, u$ and partial derivatives of $u$ up to some finite order, the action integral reads

$$
I=\int_{T} \int_{\Omega(t)} \mathscr{L} d \Omega d t
$$

where $T$ is some time interval, and the spatial domain $\Omega$ may depend on time.

Restrictions on the motion of $\Omega$ will be imposed further on, but first the Euler-Lagrange equation for $\mathscr{L}$ and natural boundary conditions are derived under the assumption that the motion of $\Omega$ is given.

For the system considered, Hamilton's principle can be summarized by saying that the evolution of the system is such that the action integral $I$ has a stationary value (see Goldstein [9]). The first variation of $I$ with respect to a variation $\eta$ is defined as

$$
\delta I(u ; \eta) \equiv\left[\frac{d}{d \varepsilon} I(u+\varepsilon \eta)\right]_{\varepsilon=0}=\int_{T} \int_{\Omega(t)} D \mathscr{L}(u) \circ \eta d \Omega d t,
$$

where

$$
D \mathscr{L}(u) \circ \eta \equiv\left[\frac{d}{d \varepsilon} \mathscr{L}(u+\varepsilon \eta)\right]_{\varepsilon=0}
$$

denotes the Fréchet derivative of $\mathscr{L}$, defined for the variation $\eta$.

To arrive at the Euler-Lagrange equation for $\mathscr{L}$, partial integrations with respect to $\vec{x}$ and $t$ are required, leaving expressions on the boundary when $\eta$ and its partial derivatives do not vanish there. So, in general, the formula for partial integration reads:

$$
D \mathscr{L}(u) \circ \eta=\delta \mathscr{L}(u) \eta+\partial_{t} \mathscr{B}_{\mathscr{L}}^{0}(u ; \eta)+\operatorname{div} \overrightarrow{\mathscr{B}}_{\mathscr{L}}^{1}(u ; \eta),
$$

where $\delta \mathscr{L}(u)$ is the variational derivative of $\mathscr{L}$ and $\mathscr{B}_{\mathscr{L}}^{0}$ and $\overrightarrow{\mathscr{B}}_{\mathscr{L}}^{1}$ are expressions linear in $\eta$.

Substitution of (4) into (2) gives

$$
\delta I(u ; \eta)=\int_{T} \int_{\Omega(t)}\left[\delta \mathscr{L}(u) \eta+\partial_{t} \mathscr{\mathscr { B }}_{\mathscr{L}}^{0}(u ; \eta)+\operatorname{div} \overrightarrow{\mathscr{B}}_{\mathscr{L}}^{1}(u ; \eta)\right] d \Omega d t .
$$

Taking into account the prescribed motion of the spatial domain $\Omega$, and ap- 
plying Gauss' divergence theorem, we can write this equation as

$$
\begin{aligned}
\delta I(u ; \eta)= & \int_{T} \int_{\Omega(t)} \delta \mathscr{L}(u) \eta d \Omega d t+\int_{T} \frac{d}{d t}\left\{\int_{\Omega(t)} \mathscr{B}_{\mathscr{L}}^{0}(u ; \eta) d \Omega\right\} d t \\
& -\int_{T} \int_{\partial \Omega(t)} \mathscr{\mathscr { B }}_{\mathscr{L}}^{0}(u ; \eta) v_{n} d S d t+\int_{T} \int_{\partial \Omega(t)} \overrightarrow{\mathscr{B}}_{\mathscr{L}}^{1}(u ; \eta) \cdot \vec{n} d S d t,
\end{aligned}
$$

where $\vec{n}$ is the outward pointing normal to $\partial \Omega$, and $v_{n}$ is the velocity of the boundary in normal direction.

If we allow $\eta$ and its partial derivatives to be arbitrary at the boundary $\partial \Omega$, but vanishing at the end points of the time interval $T$, the second term in the right-hand side vanishes (since $\mathscr{B}_{\mathscr{L}}^{0}$ is linear in $\eta$ ), and the first variation of $I$ with respect to $\eta$ is then given by

$$
\begin{aligned}
\delta I(u ; \eta)= & \int_{T} \int_{\Omega(t)} \delta \mathscr{L}(u) \eta d \Omega d t \\
& +\int_{T} \int_{\partial \Omega(t)}\left[\overrightarrow{\mathscr{B}}_{\mathscr{L}}^{1}(u ; \eta) \cdot \vec{n}-\mathscr{B}_{\mathscr{L}}^{0}(u ; \eta) v_{n}\right] d S d t
\end{aligned}
$$

From this expression the evolution of the system follows. The Euler-Lagrange equation reads

$$
\delta \mathscr{L}(u)=0 \text { in } \Omega \text {, }
$$

and the natural boundary condition on the moving boundary is given by

$$
\mathscr{B}_{\mathscr{L}}^{0}(u ; \eta) v_{n}-\overrightarrow{\mathscr{B}}_{\mathscr{L}}^{1}(u ; \eta) \cdot \vec{n}=0 \text { on } \partial \Omega,
$$

for arbitrary $\eta$.

Example. If the Lagrangian density $\mathscr{L}$ depends on $\vec{x}, t, u$ and partial derivatives up to first order only, the Fréchet derivative of $\mathscr{L}$ with respect to a variation $\eta$ reads

$$
D \mathscr{L}(u) \circ \eta=\frac{\partial \mathscr{L}}{\partial u} \eta+\frac{\partial \mathscr{L}}{\partial u_{t}} \eta_{t}+\frac{\partial \mathscr{L}}{\partial u_{x_{i}}} \eta_{x_{i}}
$$

which can be written as

$$
D \mathscr{L}(u) \circ \eta=\left[\frac{\partial \mathscr{L}}{\partial u}-\partial_{t} \frac{\partial \mathscr{L}}{\partial u_{t}}-\partial_{x_{i}} \frac{\partial \mathscr{L}}{\partial u_{x_{i}}}\right] \eta+\partial_{t}\left[\frac{\partial \mathscr{L}}{\partial u_{t}} \eta\right]+\partial_{x_{i}}\left[\frac{\partial \mathscr{L}}{\partial u_{x_{i}}} \eta\right]
$$

The first variation of $I$ is given by $(5)$, with the variational derivative of $\mathscr{L}$ being

$$
\delta \mathscr{L}(u)=\frac{\partial \mathscr{L}}{\partial u}-\partial_{t} \frac{\partial \mathscr{L}}{\partial u_{t}}-\partial_{x_{i}} \frac{\partial \mathscr{L}}{\partial u_{x_{i}}},
$$

and the expressions on the boundary being

$$
\begin{gathered}
\mathscr{B}_{\mathscr{L}}^{0}(u ; \eta)=\frac{\partial \mathscr{L}}{\partial u_{t}} \eta, \\
\overrightarrow{\mathscr{B}}_{\mathscr{L}}^{1}(u ; \eta)=\left(\frac{\partial \mathscr{L}}{\partial u_{x_{1}}}, \frac{\partial \mathscr{L}}{\partial u_{x_{2}}}, \ldots, \frac{\partial \mathscr{L}}{\partial u_{x_{n}}}\right)^{T} \eta .
\end{gathered}
$$


The vanishing of $\delta I(u ; \eta)$ yields the Euler-Lagrange equation (see (8))

$$
\frac{\partial \mathscr{L}}{\partial u}-\partial_{t} \frac{\partial \mathscr{L}}{\partial u_{t}}-\partial_{x_{i}} \frac{\partial \mathscr{L}}{\partial u_{x_{i}}}=0
$$

and the natural boundary condition (see (9))

$$
\frac{\partial \mathscr{L}}{\partial u_{t}} v_{n}-\frac{\partial \mathscr{L}}{\partial u_{x_{i}}} n_{i}=0
$$

For deriving boundary conditions (on a fixed domain of integration) that do not influence the solution on the interior of the domain, several options are available. One important a priori requirement in the development of radiation boundary conditions is that they should not be based on (properties of) solutions to the partial differential equations considered, since in general (for nonlinear equations) such solutions cannot easily be found (compare this with Higdon's derivation of absorbing boundary conditions [13], which is based on an exact plane monochromatic wave).

The possibility that will be examined in this paper is to find boundary conditions that simulate a moving domain of integration such that the energy is conserved as well as possible. The naive idea is that if the correct amount of energy is fluxed through the fixed boundary, any reflection caused by the presence of the boundary will have little energy and therefore will be small in this sense. This reasoning may be valuable, since energy density is a definite quantity. However, other densities (e.g., the momentum density) may do as well, as we shall show further on.

In the derivation above, the motion of the domain $\Omega$ was assumed to be given. Then the evolution of the system is completely determined by the EulerLagrange equation (8) and the natural boundary condition (9). From now on, we do not prescribe the motion of the domain $\Omega$, but demand instead that $\Omega$ moves in such a way that the integral over $\Omega$ of some density is conserved.

In order to show the dependence of the results on the choice of density later on, we shall consider quite generally a density for which a local conservation law can be obtained with Noether's theorem. For a comprehensive description of this well-known theorem the reader is referred to Olver [17] and the historic work of Noether [16]. The main principle of Noether's theorem can be formulated as follows (see Goldstein [9]): if the Lagrangian density is invariant under a given transformation of the variables, then there is a corresponding functional that is conserved.

So, let the Lagrangian density $\mathscr{L}$ be invariant under a variation $\varphi(u)$; then it can be proven that for some scalar density $\chi^{0}(u ; \varphi(u))$ and some vector density $\vec{\chi}^{1}(u ; \varphi(u))$ it holds that for all $u$

$$
D \mathscr{L}(u) \circ \varphi(u)=\partial_{t} \chi^{0}(u ; \varphi(u))+\operatorname{div} \vec{\chi}^{1}(u ; \varphi(u)) .
$$

A combination of this equation and the integration-by-part formula (4) yields (taking $\eta=\varphi(u)$ )

$$
\begin{aligned}
\delta \mathscr{L}(u) \varphi(u)= & \partial_{t}\left[\chi^{0}(u ; \varphi(u))-\mathscr{B}_{\mathscr{L}}^{0}(u ; \varphi(u))\right] \\
& +\operatorname{div}\left[\vec{\chi}^{1}(u ; \varphi(u))-\overrightarrow{\mathscr{B}}_{\mathscr{L}}^{1}(u ; \varphi(u))\right],
\end{aligned}
$$

leading to a local conservation law of the form

$$
\partial_{t} e(u)+\operatorname{div} \vec{f}(u)=0
$$


for solutions to the Euler-Lagrange equation (8). In this equation the density $e$ and the corresponding flux density $\vec{f}$ are given by

$$
\begin{aligned}
& e(u)=\chi^{0}(u ; \varphi(u))-\mathscr{B}_{\mathscr{L}}^{0}(u ; \varphi(u)), \\
& \vec{f}(u)=\vec{\chi}^{1}(u ; \varphi(u))-\overrightarrow{\mathscr{B}}_{\mathscr{L}}^{1}(u ; \varphi(u)) .
\end{aligned}
$$

As motivated above, let the motion of $\Omega$ be restricted in such a way that the quantity

$$
E \equiv \int_{\Omega(t)} e(u) d \Omega
$$

is conserved.

Differentiation of (22) with respect to the time gives

$$
\frac{d E}{d t}=\int_{\Omega(t)} \partial_{t} e(u) d \Omega+\int_{\partial \Omega(t)} e(u) v_{n} d S .
$$

Substitution of (19) and application of Gauss' divergence theorem yields

$$
\frac{d E}{d t}=\int_{\Omega(t)} \operatorname{div}[e(u) \vec{v}-\vec{f}(u)] d \Omega,
$$

where $\vec{v}$ represents a velocity field on $\Omega$ such that

$$
v_{n}=\vec{v} \cdot \vec{n}
$$

on the boundary $\partial \Omega$.

If the quantity $E$ is conserved, then $\vec{v}$ must satisfy

$$
\int_{\Omega(t)} \operatorname{div}[e(u) \vec{v}-\vec{f}(u)] d \Omega=0 .
$$

Since the initial domain $\Omega$ can be taken arbitrarily, $\vec{v}$ necessarily equals the local flux velocity $\vec{v}_{f}$, defined by

$$
\vec{f}(u)=\vec{v}_{f} e(u) .
$$

So, in particular, the normal velocity $v_{n}$ on the boundary equals the local flux velocity in normal direction,

$$
v_{n}=\vec{v}_{f} \cdot \vec{n}=\frac{\vec{f}(u) \cdot \vec{n}}{e(u)},
$$

at points where $e(u)$ does not vanish. The latter condition can also be obtained directly from (23); substitution of (19) and application of Gauss' divergence theorem gives

$$
\frac{d E}{d t}=\int_{\partial \Omega(t)}\left[e(u) v_{n}-\vec{f}(u) \cdot \vec{n}\right] d S .
$$

If the quantity $E$ is conserved, then $v_{n}$ is determined by

$$
e(u) v_{n}-\vec{f}(u) \cdot \vec{n}=0,
$$

in accordance with (28).

Remark 1. Substitution of (27) into (19) gives

$$
\partial_{t} e(u)+\vec{v}_{f} \cdot \nabla e(u)+e(u) \operatorname{div} \vec{v}_{f}=0,
$$


which in case of a divergence-free flux velocity field $\vec{v}_{f}$ reduces to

$$
\frac{D e(u)}{D t}=\partial_{t} e(u)+\vec{v}_{f} \cdot \nabla e(u)=0,
$$

expressing that $e(u)$ is constant along the streamlines of the corresponding flux velocity field $\vec{v}_{f}$.

With the explicit expressions (20) and (21) for $e$ and $\vec{f}$, respectively, condition (30) reads

$$
\left[\chi^{0}(u ; \varphi(u))-\mathscr{B}_{\mathscr{L}}^{0}(u ; \varphi(u))\right] v_{n}-\left[\vec{\chi}^{1}(u ; \varphi(u))-\overrightarrow{\mathscr{B}}_{\mathscr{L}}^{1}(u ; \varphi(u))\right] \cdot \vec{n}=0 .
$$

However, the natural boundary condition (9) already implies $(\eta=\varphi(u))$

$$
\mathscr{B}_{\mathscr{L}}^{0}(u ; \varphi(u)) v_{n}-\overrightarrow{\mathscr{B}}_{\mathscr{L}}^{1}(u ; \varphi(u)) \cdot \vec{n}=0,
$$

and so the additional boundary condition for conservation of the quantity $E$ is given by

$$
\chi^{0}(u ; \varphi(u)) v_{n}-\vec{\chi}^{1}(u ; \varphi(u)) \cdot \vec{n}=0,
$$

where $\chi^{0}(u ; \varphi(u))$ and $\vec{\chi}^{1}(u ; \varphi(u))$ are obtained from (17).

Summarizing, the proposed boundary conditions for a domain $\Omega$ that is chosen in such a way that the total amount of a density $e$ is preserved, are given by the natural boundary condition (9) and the additional boundary condition (35).

Remark 2. If $e$ equals the energy density, then the local flux velocity $\vec{v}_{f}$ defined by (27) is well known, in particular for linear systems, for which it equals the group velocity for monochromatic waves; see, e.g., Biot [3], Broer [4], and Lighthill [15]. It is the pointwise version of the centro velocity (i.e., the velocity of the center of gravity of the density $e$ ), which is given by

$$
\vec{v}_{C}=\frac{\vec{F}}{E}
$$

where $\vec{F} \equiv \int_{\Omega} \vec{f}(u) d \Omega$ and $E \equiv \int_{\Omega} e(u) d \Omega$ (see van Groesen [10], van Groesen and Mainardi [11], and Wehausen and Laitone [19]).

Remark 3. If $e$ equals the energy density, then the ideas above are related to some parts of the earlier work of Whitham (see [20, Chapter 11]). In his treatment of energy propagation for the Klein-Gordon equation a slowly varying wave train is considered. It is found that the averaged energy density $\mathscr{E}$ and the averaged energy flux density $\mathscr{F}$ (i.e., averaged over one period) satisfy

$$
\mathscr{F}=C(k) \mathscr{E},
$$

where $C(k)$ denotes the group velocity depending on the wave number $k$. Compare this result with the definition of the local flux velocity in (27).

Next an averaged energy equation is proposed:

$$
\partial_{t} \mathscr{E}+\partial_{x}(C \mathscr{E})=0
$$

which is the differential form of the statement that

The total energy between any two group lines remains constant. 
Evidently, (38) in combination with (37) can be regarded as a global conservation law. Compare this with our local conservation law (19). Note that the present derivation of radiation boundary conditions started from a similar, yet more general, principle, namely the conservation of an appropriate density on a moving domain of integration.

Example. If the first-order Lagrangian density $\mathscr{L}$ does not depend explicitly on $\vec{x}$ and $t$, then the energy density can be found with Noether's theorem from a variation corresponding to the invariance of $\mathscr{L}$ with respect to time translations,

$$
\varphi(u)=u_{t} .
$$

Similarly, the momentum density in some direction $\vec{\sigma}$ is obtained with a variation corresponding to the invariance of $\mathscr{L}$ with respect to spatial translations,

$$
\psi(u)=\nabla u \cdot \vec{\sigma} \quad(\vec{\sigma} \neq \overrightarrow{0}) .
$$

Substitution of $\eta=\varphi(u)$ into (10) yields

$$
D \mathscr{L}(u) \circ u_{t}=\frac{\partial \mathscr{L}}{\partial u} u_{t}+\frac{\partial \mathscr{L}}{\partial u_{t}} u_{t t}+\frac{\partial \mathscr{L}}{\partial u_{x_{i}}} u_{t x_{i}}=\partial_{t} \mathscr{L}(u),
$$

and therefore (17) holds with

$$
\begin{aligned}
& \chi^{0}(u ; \varphi(u))=\mathscr{L}(u), \\
& \vec{\chi}^{1}(u ; \varphi(u))=\overrightarrow{0} .
\end{aligned}
$$

Then, indeed, substitution of (13) and (42) into (20) yields the energy density

$$
e(u)=\mathscr{L}(u)-\frac{\partial \mathscr{L}}{\partial u_{t}} u_{t},
$$

with energy flux density (see (14), (43), and (21))

$$
\vec{f}_{e}(u)=-\left(\frac{\partial \mathscr{L}}{\partial u_{x_{1}}}, \frac{\partial \mathscr{L}}{\partial u_{x_{2}}}, \ldots, \frac{\partial \mathscr{L}}{\partial u_{x_{n}}}\right)^{T} u_{t} .
$$

Similarly, substitution of $\eta=\psi(u)$ into (10) gives

$$
\begin{aligned}
D \mathscr{L}(u) \circ(\nabla u \cdot \vec{\sigma}) & =\frac{\partial \mathscr{L}}{\partial u}(\nabla u \cdot \vec{\sigma})+\frac{\partial \mathscr{L}}{\partial u_{t}}(\nabla u \cdot \vec{\sigma})_{t}+\frac{\partial \mathscr{L}}{\partial u_{x_{i}}}(\nabla u \cdot \vec{\sigma})_{x_{i}} \\
& =\vec{\sigma} \cdot \nabla \mathscr{L}(u)=\operatorname{div}(\vec{\sigma} \mathscr{L}(u)),
\end{aligned}
$$

and (17) holds with

$$
\begin{aligned}
& \chi^{0}(u ; \psi(u))=0, \\
& \vec{\chi}^{1}(u ; \psi(u))=\vec{\sigma} \mathscr{L}(u) .
\end{aligned}
$$

The momentum density $m$ (in the direction $\vec{\sigma}$ ) reads

$$
m(u)=-\frac{\partial \mathscr{L}}{\partial u_{t}}(\nabla u \cdot \vec{\sigma}),
$$

with momentum flux density

$$
\vec{f}_{m}(u)=\vec{\sigma} \mathscr{L}(u)-\left(\frac{\partial \mathscr{L}}{\partial u_{x_{1}}}, \frac{\partial \mathscr{L}}{\partial u_{x_{2}}}, \ldots, \frac{\partial \mathscr{L}}{\partial u_{x_{n}}}\right)^{T}(\nabla u \cdot \vec{\sigma}) .
$$


Substitution of (42) and (43) into (35) yields the additional boundary condition in case of energy conservation,

$$
\mathscr{L}(u) v_{n}=0 .
$$

Similarly, the additional boundary condition in case of momentum conservation reads

$$
\mathscr{L}(u)(\vec{\sigma} \cdot \vec{n})=0 .
$$

Consequently, if we take

$$
\mathscr{L}(u)=0
$$

on the boundary $\partial \Omega$, it follows that both energy and momentum (in any direction) are conserved in this special case!

Remark 4. In [21], Whitham considers dispersive wave problems in which the Euler-Lagrange equation has approximate solutions of the form

$$
u=U(\theta, a), \quad \theta=k x-\omega t,
$$

where, in the nonlinear case, the amplitude $a$, the wave number $k$ and the frequency $\omega$ will not be constant; $k$ and $\omega$ are generalized by defining them as

$$
k=\frac{\partial \theta}{\partial x}, \quad \omega=-\frac{\partial \theta}{\partial t} .
$$

It is assumed that $\omega, k$, and $a$ are slowly-varying functions of $x$ and $t$, corresponding to the slow modulation of the wave train considered. The averaged Lagrangian $\bar{L}$ is then defined as

$$
\bar{L}(\omega, k, a)=\frac{1}{2 \pi} \int_{0}^{2 \pi} \mathscr{L} d \theta
$$

and is calculated by substituting the uniform periodic solution $u=U(\theta, a)$ in $\mathscr{L}$.

The equations for $\omega, k$, and $a$ are then obtained from the averaged variational principle

$$
\delta \iint \bar{L}(\omega, k, a) d t d x=0 .
$$

In the linear case we have

$$
\bar{L}(\omega, k, a)=G(\omega, k) a^{2},
$$

and a variation of $\bar{L}$ with respect to $a$ yields the linear dispersion relation

$$
G(\omega, k)=0 \text {. }
$$

It is then observed that the stationary value of $\bar{L}$ is zero. Compare this global condition (under the assumptions of slowly-varying wave trains and periodic solutions) to the pointwise boundary condition (53).

From now on it is assumed that $e$ equals the energy density of the system considered. In consequence, we shall use condition (51) in the next section for specific choices of $\mathscr{L}$.

\section{APPLICATION TO ONE-DIMENSIONAL WAVE EQUATIONS}

In this section we shall apply the theory from the previous section to a nonlinear one-dimensional wave equation. The boundary conditions obtained will be 
used to simulate "open" boundaries, i.e., boundaries which give low reflections for radiating waves.

Consider a nonlinear version of the Klein-Gordon equation,

$$
u_{t t}=c^{2} u_{x x}-V^{\prime}(u) \text {, }
$$

where $V^{\prime}(u)$ is some nonlinear function of $u$, and $c$ is the wave velocity. This hyperbolic equation arises in various physical problems. For example, if $V^{\prime}(u)=\sin (u)$, then $(60)$ is known as the Sine-Gordon equation.

The action integral for this problem is given by

$$
I=\int_{T} \int_{x_{0}}^{x_{1}} \mathscr{L}(u) d x d t,
$$

where the Lagrangian density $\mathscr{L}$ is a function of $u$ and its first-order derivatives (note that $\mathscr{L}$ does not depend explicitly on $t$ ):

$$
\mathscr{L}(u)=\frac{1}{2}\left(u_{t}^{2}-c^{2} u_{x}^{2}\right)-V(u) .
$$

In this paper we shall consider a potential $V(u)$ given by

$$
V(u)=\frac{1}{2} \alpha u^{2}+\frac{1}{4} \beta u^{4},
$$

which is an appropriate expansion for even functions $V$. The choice $\alpha=1$, $\beta=-1 / 3$ yields a second-order expansion for the Sine-Gordon equation. In our test cases, however, we shall restrict $\alpha$ and $\beta$ to positive values to guarantee the positive definiteness of the energy density, which for this problem reads (see (44) and (62))

$$
e(u)=\frac{1}{2}\left(u_{t}^{2}+c^{2} u_{x}^{2}\right)+V(u) .
$$

The natural boundary condition for this system is obtained from (16):

$$
c^{2} u_{n}+v_{n} u_{t}=0 \text {, }
$$

and (51) yields the additional boundary condition

$$
\left[\frac{1}{2}\left(u_{t}^{2}-c^{2} u_{x}^{2}\right)-V(u)\right] v_{n}=0 .
$$

From (65) and (66) it can be concluded that either $v_{n}=u_{n}=0$ must hold or

$$
v_{n}=c\left(-\frac{c u_{n}}{u_{t}}\right)
$$

where $u_{t}$ and $u_{n}$ are related by $\mathscr{L}=0$, i.e.,

$$
u_{t}^{2}-c^{2} u_{x}^{2}=2 V(u) \text {. }
$$

The sign of $v_{n}$ has to be chosen so as to make sure that no energy flows into the spatial domain, since energy inflow might spoil the stability of the problem; if an increasing spatial domain is simulated, and conservation of energy is required on this domain, no energy inflow will occur on the fixed domain $\Omega$. It is for this reason that $v_{n}$ is chosen positive. From (65) it can be seen that this can be enforced by requiring

$$
u_{n} u_{t} \leq 0 \text {. }
$$

If $\beta=0$, then $(60)-(63)$ reduce to the linear Klein-Gordon equation,

$$
u_{t t}=c^{2} u_{x x}-\alpha u \text {. }
$$


Solutions to (70) can be written as

$$
u=a e^{i \theta}, \quad \theta(x, t)=k x-\omega t,
$$

where $k$ is the wave number and $\omega$ is the frequency.

Substitution of (71) into (70) yields the (linear) dispersion relation

$$
\omega^{2}=c^{2} k^{2}+\alpha,
$$

from which we may calculate the group velocity

$$
c_{g} \equiv \frac{d \omega}{d k}=\frac{c^{2} k}{\sqrt{c^{2} k^{2}+\alpha}}=\frac{c^{2} k}{\omega} .
$$

Substitution of (71) into (67) gives ( $n$ is taken in the positive $x$-direction)

$$
v_{n}=\frac{c^{2} k}{\omega},
$$

and we obtain a result we might have expected for the linear case; conservation of energy on a moving domain leads to a boundary velocity which equals the group velocity.

Finally, we note that if both $\alpha$ and $\beta$ are equal to zero, condition (68) reduces to Sommerfeld's radiation condition (see Orlanski [18])

$$
u_{t}=-c u_{n}
$$

corresponding to a boundary velocity which equals the local phase velocity $c_{f}$,

$$
v_{\text {Sommerfeld }}=\frac{\omega}{c^{2} k} \equiv c_{f} \text {. }
$$

In the next section we shall use boundary conditions (68) and (75) as radiation boundary conditions in several test cases.

\section{NUMERICAL RESULTS}

For the application described in the previous section some numerical tests have been carried out. In this section the results from these numerical tests are presented and discussed.

The field equation is given by the nonlinear Klein-Gordon equation,

$$
u_{t t}=u_{x x}-\alpha u-\beta u^{3} \quad(\alpha, \beta \geq 0) \text {. }
$$

Three-point central discretizations have been used for the second-order derivatives in (77). An explicit Euler scheme has been used here, which is stable if the time step is chosen small enough.

The spatial domain and the time domain are given by

$$
\begin{aligned}
& \Omega=[-\pi, \pi], \\
& T=[0,4 \pi] .
\end{aligned}
$$

The initial conditions read

$$
\begin{aligned}
u(x, 0) & = \begin{cases}\cos ^{2}(x), & |x| \leq \pi / 2 \\
0, & |x|>\pi / 2\end{cases} \\
\frac{\partial u}{\partial t}(x, 0) & = \begin{cases}\sqrt{1+\alpha} \sin (2 x), & |x| \leq \pi / 2 \\
0, & |x|>\pi / 2\end{cases}
\end{aligned}
$$


These conditions represent a wave travelling to the right. It is obvious that (80)-(81) does not represent a steady wave for (77). In the small-amplitude limit, this initial condition may be regarded as a superposition of many monochromatic solutions, each of them travelling at a different phase velocity. Therefore, it can be expected that from the very beginning some energy will propagate to the left. This means that the radiation boundary conditions have to be implemented in both endpoints.

The new radiation boundary condition is given by

$$
u_{t}=-u_{n} \sqrt{1+\frac{\alpha u^{2}+(\beta / 2) u^{4}}{u_{n}^{2}}}
$$

and Sommerfeld's radiation condition reads

$$
u_{t}=-u_{n} \text {. }
$$

Condition (82) can be written as

$$
u_{t}=-c(u) u_{n},
$$

i.e., a Sommerfeld radiation condition (see (75)) where the correction factor (or velocity) $c(u)$ is given by

$$
c(u)=\sqrt{1+\frac{\alpha u^{2}+(\beta / 2) u^{4}}{u_{n}^{2}}} .
$$

If $u_{n}$ approaches zero, which corresponds to a local extremum of the solution $u$ near the boundary, then (82) would give a significant value for $u_{t}$, involving an undesirable change in $u$ at the boundary. Therefore, we set an upper limit to the correction velocity:

$$
c^{*}(u)=\min (c(u), \sqrt{1+\alpha}) .
$$

The new radiation boundary condition then reads

$$
u_{t}=-c^{*}(u) u_{n} .
$$

Since we have chosen an explicit discretization of the field equation, we have implemented boundary conditions (87) and (83) explicitly too.

For lack of analytical solutions to (77) with initial conditions $(80)-(81)$, the numerical solutions obtained with (87) and (83) will be compared with the solution obtained on an extended spatial domain $\Omega^{\prime} \supset \Omega$. It is important that $\Omega^{\prime}$ is chosen large enough, so that reflections from the boundaries of $\Omega^{\prime}$ do not reach the smaller domain $\Omega$ during the chosen time interval $T$. Test results show that this requirement is met by the choice

$$
\Omega^{\prime}=[-3 \pi, 3 \pi] \text {. }
$$

On this extended domain the initial conditions (80)-(81) are imposed. Since the numerical errors on the interior of $\Omega$ are equal to those on the interior of $\Omega^{\prime}$, we get a good indication of the errors due to the radiation boundary conditions.

Three configurations have been used for the calculation of the numerical solution to (77) with initial conditions $(80)-(81)$ :

- Configuration 1: the numerical solution is determined on the spatial domain $\Omega$ with the new radiation boundary condition (87) implemented 
in the endpoints $x= \pm \pi$, where the correction velocity $c^{*}(u)$ is determined by (86) and (85).

- Configuration 2: the numerical solution is determined on the spatial domain $\Omega$ with Sommerfeld's radiation condition (83) implemented in the endpoints $x= \pm \pi$.

- Configuration 3: the numerical solution is determined on the extended spatial domain $\Omega^{\prime}$ with Sommerfeld's radiation boundary condition implemented in the endpoints $x= \pm 3 \pi$. In this way we obtain an "undisturbed" (i.e., not disturbed by artificial boundaries) numerical solution on the smaller domain $\Omega$, which can be used to calculate the errors due to the radiation boundary conditions in Configurations 1 and 2.

In order to get an indication of the error $\varepsilon=u-\tilde{u}$ ( $\tilde{u}$ denotes the numerical approximation to $u$ ), we use two norms. The first norm is the $L_{2}$-norm of $\varepsilon$, which is defined by

$$
\|\varepsilon\|_{2}=\left\{\int_{\Omega} \varepsilon^{2}(x, t) d x\right\}^{1 / 2} .
$$

The second norm is an energy norm of $\varepsilon$,

$$
\|\varepsilon\|_{E}=\int_{\Omega} \frac{1}{2}\left(\varepsilon_{t}^{2}+\varepsilon_{x}^{2}\right) d x .
$$

Five tests have been performed for different combinations of $\alpha$ and $\beta$. In the first three tests, $\beta$ is equal to zero, yielding a linear field equation. However, note that boundary condition (87) (Configuration 1) is nonlinear if either $\alpha$ or $\beta$ is not equal to zero. In the last two tests the values of both $\alpha$ and $\beta$ are nonzero, yielding a nonlinear field equation.

Test 1: $\alpha=0.1, \beta=0.0$. In this case the (linear) disturbance in (77) is rather small. Figure $1^{1}$ shows the $L_{2}$-norm of the errors in the solutions obtained with Configurations 1 and 2. In Figure 2 the energy norm of the errors is presented. The errors obtained with the new radiation boundary condition are about the same as the errors obtained with Sommerfeld's radiation condition. An explanation is given by (85): if both $\alpha$ and $\beta$ are small, then the correction factor will be but slightly larger than one.

It is convenient to combine Figures 3 and 4. Figure 3 shows the energy of the numerical solution obtained with the first configuration during the first two periods. In this graph the parts of rapid decrease in energy correspond to high gradients in the numerical solution passing the right boundary $x=\pi$ (see Figure 4). Similarly, the inflection point at $t=\pi$ corresponds to the crest of the wave arriving at the right boundary.

Test 2: $\alpha=1.0, \beta=0.0$. Because of the larger value of $\alpha$, which implies a stronger (linear) disturbance, the errors are expected to be larger than in Test 1. This is confirmed in Figures 5 and 6, which show the $L_{2}$-norm and the energy norm of the errors, respectively.

For $0 \leq t \leq 3$ the errors obtained with the first configuration are somewhat larger than the errors obtained with Configuration 2. For $t>3$ there is a

\footnotetext{
${ }^{1}$ In Figures $1-12$ a dashed line equals boundary condition (83) and a solid line equals boundary condition (87).
} 


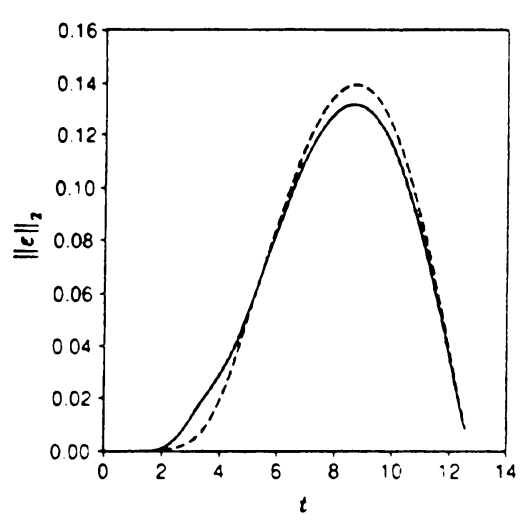

FIGURE 1

Test $1: \alpha=0.1, \beta=0.0$.

$L_{2}$-norm of error

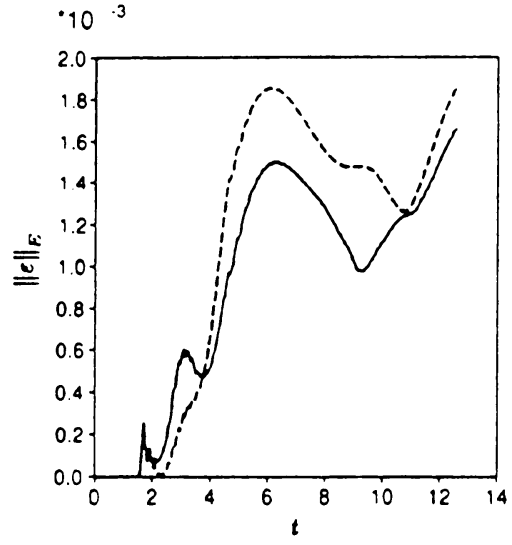

FIGURE 2

Test $1: \alpha=0.1, \beta=0.0$.

Energy norm of error

$$
\begin{aligned}
\text { dashed line } & =\text { boundary condition }(83) \\
\text { solid line } & =\text { boundary condition }(87)
\end{aligned}
$$

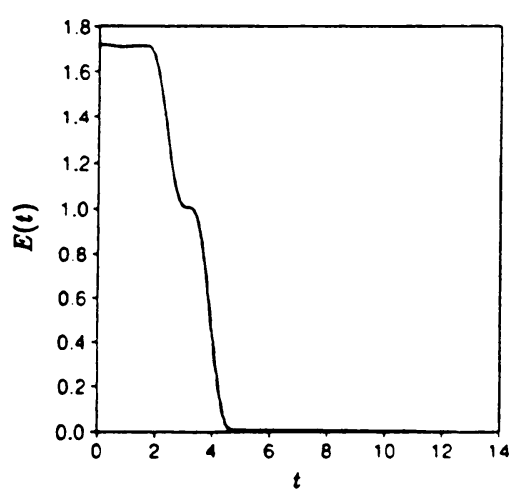

FIGURE 3

Test $1: \alpha=0.1, \beta=0.0$,

Configuration 1. Energy of solution

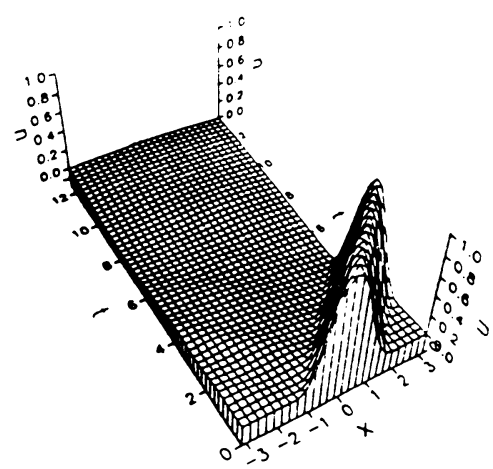

FIGURE 4

Test $1: \alpha=0.1, \beta=0.0$, Configuration 1. Solution $u$

clear advantage of the first configuration over the second. Obviously, $\alpha$ is large enough to make the correction velocity $c^{*}(u)$ in (87) effective. After two periods, the energy norms of the errors obtained with Configurations 1 and 2 are equal to 0.01 and 0.03 , respectively. 


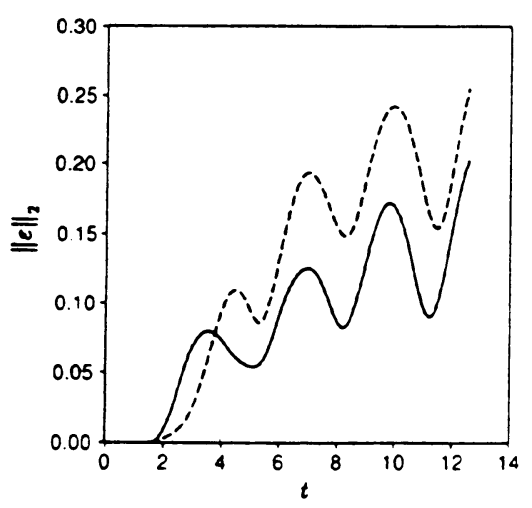

FIGURE 5

Test $2: \alpha=1.0, \beta=0.0$.

$L_{2}$-norm of error

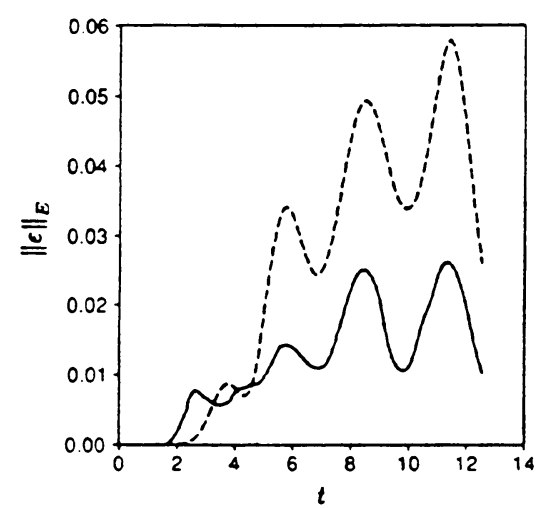

FigURE 6

Test $2: \alpha=1.0, \beta=0.0$.

Energy norm of error

$$
\begin{aligned}
\text { dashed line } & =\text { boundary condition }(83) \\
\text { solid line } & =\text { boundary condition }(87)
\end{aligned}
$$

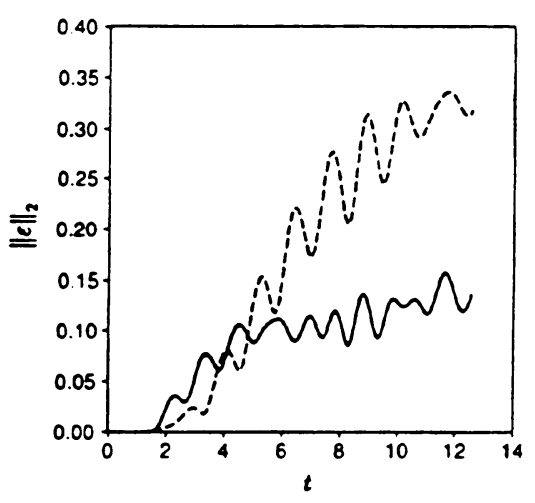

FIGURE 7

Test $3: \alpha=5.0, \beta=0.0$.

$L_{2}$-norm of error

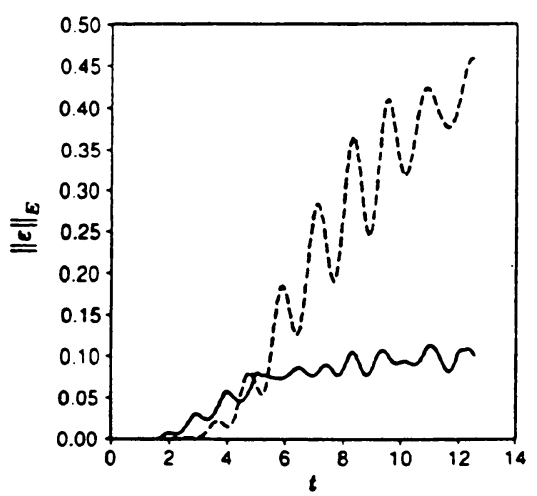

FIGURE 8

Test $3: \alpha=5.0, \beta=0.0$.

Energy norm of error

Test 3: $\alpha=5.0, \beta=0.0$. Figures 7 and 8 show a great advantage of the new radiation boundary condition over Sommerfeld's radiation condition. After two periods the $L_{2}$-norms of the errors obtained with Configurations 1 and 2 are equal to 0.13 and 0.32 , respectively. The energy norms of the errors obtained are equal to 0.10 (Configuration 1) and 0.45 (Configuration 2). 


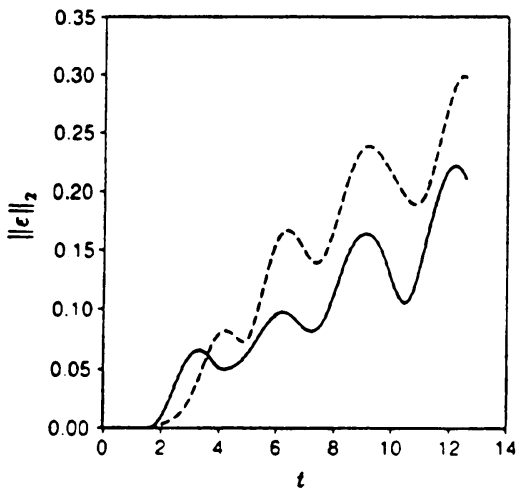

FIGURE 9

Test $4: \alpha=1.0, \beta=0.5$.

$L_{2}$-norm of error

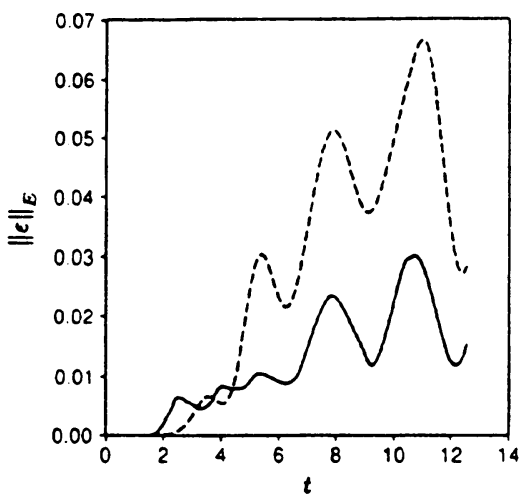

FIGURE 10

Test $4: \alpha=1.0, \beta=0.5$.

Energy norm of error

$$
\begin{aligned}
\text { dashed line } & =\text { boundary condition }(83) \\
\text { solid line } & =\text { boundary condition }(87)
\end{aligned}
$$

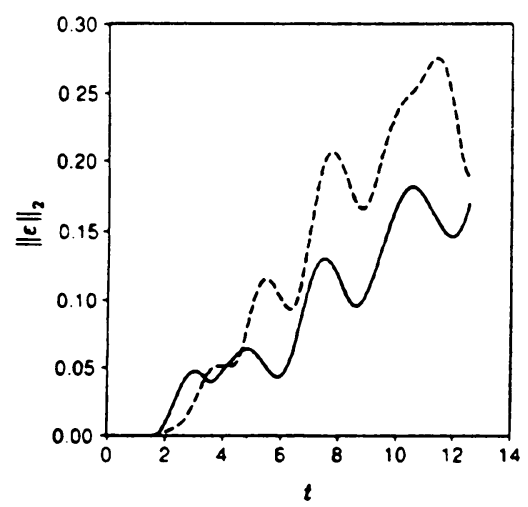

FIGURE 11

Test $5: \alpha=1.0, \beta=2.0$.

$L_{2}$-norm of error

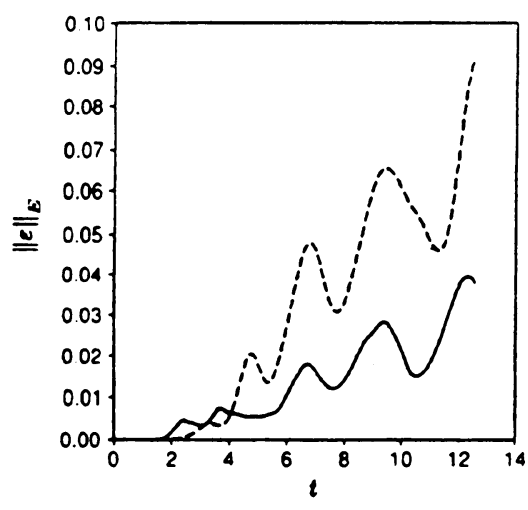

FIGURe 12

Test $5: \alpha=1.0, \beta=2.0$.

Energy norm of error

It is clear that the new radiation boundary condition provides better results for larger values of $\alpha$. As mentioned before, this is due to the correction factor $c^{*}(u)$ in $(87)$. 
In the last two tests we shall investigate the influence of a nonlinear disturbance in the field equation on the performance of boundary condition (87).

Test 4: $\alpha=1.0, \beta=0.5$. Since $\beta$ is not equal to zero, the field equation (77) is nonlinear. The results obtained with Configuration 1 are better than those obtained with Configuration 2, as can be seen in Figures 9 and 10. The $L_{2}$-norms after two periods are equal to 0.21 (Configuration 1) and 0.30 (Configuration 2 ). The maximum values of the energy norm are equal to 0.02 (Configuration 1) and 0.03 (Configuration 2).

Note that for both configurations the errors are but slightly larger than in Test $2(\alpha=1.0, \beta=0.0)$ (cf. Figures 5-6). This indicates a dominant linear term $\alpha u$ in the disturbance in the field equation for this combination of $\alpha$ and $\beta$. Therefore, in the next test we shall increase the impact of the nonlinear term $\beta u^{3}$ by raising the value of $\beta$.

Test 5: $\alpha=1.0, \beta=2.0$. The expected growth in the errors (see Test 4) can only be found in Figure 12, which shows the energy norm of the errors. The $L_{2}$-norm of the error is (for both configurations) of the same order as in Test $2(\beta=0.0)$. This indicates a stronger variation in the derivatives of the errors owing to the stronger nonlinear disturbance (see (90)).

For this combination of $\alpha$ and $\beta$, Configuration 1 provides better results than Configuration 2 (see Figures 11 and 12). The $L_{2}$-norms of the errors after two periods are equal to 0.17 (Configuration 1) and 0.19 (Configuration 2). The average values, however, show better results for Configuration 1 than for Configuration 2. The energy norm after two periods is equal to 0.04 (Configuration 1) and 0.09 (Configuration 2).

\section{CONCLUSIONS}

A new method has been presented for developing radiation boundary conditions for systems governed by a Lagrangian variational principle. These boundary conditions are based on conservation laws for appropriate densities on a moving domain of integration. The main advantage of this method over other methods is that it is not based on (properties of) solutions to the field equation considered, and therefore it is valid for nonlinear and dispersive systems too. Moreover, the boundary conditions obtained render the problem well posed in the sense that the integrated density is conserved.

An explicit boundary condition has been derived for a nonlinear version of the Klein-Gordon equation, based on considerations for the energy. The numerical test results show a better performance of this new radiation boundary condition in comparison with Sommerfeld's radiation condition.

For an application of the theory to the two-dimensional wave equation the reader is referred to [5].

\section{BIBLIOGRAPHY}

1. A. Bayliss and E. Turkel, Radiation boundary conditions for wave-like equations, Comm. Pure Appl. Math. 33 (1980), 707-725.

2. $\ldots$, Far field boundary conditions for compressible flows, J. Comput. Phys. 48 (1982), 182-199. 
3. M. A. Biot, General theorem on the equivalence of group velocity and energy transport, Phys. Rev. 105 (1957), 1129-1137.

4. L. J. F. Broer, On the propagation of energy in linear conservative waves, Appl. Sci. Res. A (1951), 329-344.

5. J. Broeze and E. F. G. van Daalen, Radiation boundary conditions for the two-dimensional wave equation from a variational principle, Math. Comp. 58 (1992), 73-82.

6. B. Engquist and L. Halpern, Far field boundary conditions for computation over long time, Appl. Numer. Math. 4 (1988), 21-45.

7. B. Engquist and A. Majda, Absorbing boundary conditions for the numerical simulation of waves, Math. Comp. 31 (1977), 629-651.

8. Radiation boundary conditions for acoustic and elastic wave calculations, Comm. Pure Appl. Math. 32 (1979), 313-357.

9. H. Goldstein, Classical mechanics, Addison-Wesley, Reading, MA, 1980.

10. E. W. C. van Groesen, Unidirectional wave propagation in one dimensional, first order Hamiltonian systems, J. Math. Phys. 21 (1980), 1646-1655.

11. E. van Groesen and F. Mainardi, Balance laws and centro velocity in dissipative systems, J. Math. Phys. 31 (1990), 2136-2140.

12. R. L. Higdon, Absorbing boundary conditions for difference approximations to the multidimensional wave equation, Math. Comp. 47 (1986), 437-459.

13. __ Numerical absorbing boundary conditions for the wave equation, Math. Comp. 49 (1987), 65-90.

14. __ Radiation boundary conditions for elastic wave propagation, SIAM J. Numer. Anal. 27 (1990), 831-870.

15. M. J. Lighthill, Group velocity, J. Inst. Math. Appl. 1 (1965), 1-28.

16. E. Noether, Invariante Variationsprobleme, Nachr. Gesell. Wiss. Göttingen 2 (1918), 235257.

17. P. J. Olver, Application of Lie groups to differential equations, Springer-Verlag, New York, 1986.

18. I. Orlanski, A simple boundary condition for unbounded hyperbolic flows, J. Comput. Phys. 21 (1976), 251-269.

19. J. V. Wehausen and E. V. Laitone, Surface waves, Fluid Dynamics III, Vol. IX, Surface Waves, Encyclopedia of Physics, Springer-Verlag, Berlin, 1960, pp. 446-778.

20. G. B. Whitham, Linear and nonlinear waves, Wiley, New York, 1974.

21. __ Two-timing, variational principles and waves, J. Fluid Mech. 44 (1970), 373-395.

Maritime Research INStitute Netherlands (MARIN), P.O. Box 28, 6700 AA WageniNGEN, THE NETHERLANDS

Delft Hydraulics, P.O. Box 152, 8300 aD Emmeloord, The Netherlands

University of Twente, P.O. BoX 217, 7500 AE Enschede, The Netherlands 